\title{
Impact of Rods Configuration on Electrodes Impedance of Through-the-Earth Communication System
}

\author{
Jianjun Hao ${ }^{1}$, Yongfei Mou ${ }^{1}$ and Bin Yan $^{1}$ \\ ${ }^{1}$ School of Electronics, Communications \& physics Shandong University of \\ Science \& Technology, Qingdao 266590, China \\ skdjjhao@163.com,279033894@qq.com,yanbinhit@hotmail.com
}

\begin{abstract}
Through-The-Earth (TTE) systems are usually applied in mining and cave rescues. In order to enlarge the communication range of TTE system with electrodes, the injection current intensity must be increased, and minimizing the impedance between electrodes is an efficient way to extend the transmission distance of the system. Aiming at decreasing the volume of electrodes resistance, several multi-rods electrode schemes are provided and implemented to test the impacts of rods amount, rod buried depth and arrangement on trans-impedance of electrodes. Experimental results illustrate that the electrodes resistance can be efficiently minimized by increasing the number of rods, and the electrodes impedance decreases with rod buried depth increase. Through unsuitable for underground implementation, rods configured in square or circle is slightly better than configured in line in reducing the electrodes resistance. However circle or square configuration is not suitable for underground. The experiments also demonstrate that the earth conductivity is an essential factor in TTE communications.
\end{abstract}

Keywords: through-the-earth communication, electrodes impedance, multi-rod, earth conductivity

\section{Introduction}

Underground mining is undertaken in a harsh environment, and sometimes the miners may experience risks such as collapse, explosion and fire. These accidents can cause damage to the existing communication channels, and further disable the on-operating communication systems. Once it occurs, an emergent system to to guarantee clear and constant communication between trapped miners and the rescue center on the ground would be necessitated. Due to its stable channel, a through-the-earth (TTE) communication system can serve as an emergent communication means.

There are two main TTE communication techniques: inductive coupling by means of loop antennas and current injection with electrodes. Currently induction coupling method is adopted by most of the TTE system, such as PED system developed by MST Company of Australia, and Magnelink system developed by Lockheed-Martin Corporation of U.S. However there are still many constraints for this technique, such as medium absorption and skin effect while electromagnetic wave propagating in the earth, and they may cause a large attenuation. Since the propagation attenuation becomes worse with the rising of signal frequency, so TTE systems always operate at a low frequency. Lower frequency result in a larger antenna, therefore large size loop antenna acts as another constraint for TTE communication system of inductive coupling.

TTE system with electrodes sends signals by injecting AC current to the earth by a pair of electrodes, and that current signals are then detected by another pair of electrodes in the receiver end. It is more efficient than inductive coupling scheme with small loop antenna [1]. But TTE systems with electrodes also have its problem of the large variation range of load impedance (impedance between electrodes). If the earth impedance between the two 
electrodes is more than $100 \Omega$, then the output power would be restricted at a very low level, and further the communication distance will decreased tremendously. How to reduce electrodes impedance now becomes an important issue in extending the communication range of TTE system with electrodes.

\section{Related Works}

Since the larger volume of current injected into the earth leads to the larger communication range, reducing the impedance between electrodes seems to be an option under the condition of limited power. In literature [2] an apparent earth conductivity estimates were made based on the homogeneous half-space earth model to estimate the earth conductivity. By this model, earth impedance can be computed, but the estimates model wasn't verified by experiments. In literature [3-5], Experimental studies on contact impedance measurements were conducted with different electrodes, such as steel L-bar electrode, copper braid electrode and Aluminum foil electrodes and a variety of contact conditions, also the best medium access is proved to be a copper braid with bentonite by Bataller in Ref. [6]. But in the experiments performed by Bataller et al., the electrodes span and the transmitter-receiver distance are not long enough to verify its performance in a true transmission environment. In literature [7] a predictive model for magnetic field distribution was proposed and also the impact of overburden conductivity is analyzed by L. Yan et al.

In this work, we provide a multi-rod electrode scheme, and then perform a series of experiments in a coal mine and a copper mine, on ground and underground to test the impacts of the rods number and its arrangement on impedance between electrodes and transmitter output power.

\section{Model of TTE Communications}

In TTE communication systems with electrodes, the transmitter injects a current into the earth by supplying a voltage between electrodes. The electrical voltage is controlled by the signal to yield an alternating current in the earth, and then this signal is captured by another pair of electrodes in the receiver end.

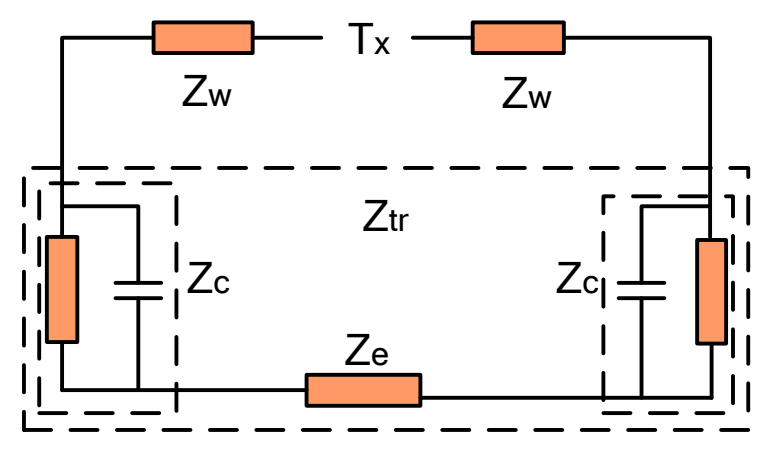

Figure 1. Equivalent Circuit Model of Transmitter at ELF

Output injection current intensity depends on the impedance and the voltage between electrodes. Figure 1 is the simplified equivalent circuit model seen by transmitter output stage. In Fig. $1, Z_{t r}$ is the impedance between electrodes, which consisted of $Z_{c}$ and $Z_{e}$; $Z_{e}$ is the earth medium impedance, $Z_{c}$ is the contact impedance between the earth and electrode, and $Z_{w}$ is the wire impedance. To improve the injection current intensity, a method is to reduce the load impedance including wire impedance and electrodes impedance. Also the current injected into the earth is determined by the transmitter output voltage $U$, intrinsic impedance $Z_{0}$ in transmitter, together with wire impedance $Z_{W}$ and 
electrodes impedance $Z_{t r}$, i.e., $I=U /\left(Z_{0}+Z_{W}+Z_{t r}\right)$, where $I$ is the injection current intensity. The intrinsic impedance of transmitter is non-adjustable (usually less than $20 \Omega$ ), and the wire impedance is far less than electrodes impedance, so reducing the electrodes impedance $Z_{t r}$ can result in an evidently increased injection current intensity.

In order to reduce the electrodes impedance, a multi-rods (or braids) electrode scheme is provided as an efficient method to minimize the electrodes impedance since multi-rods can enlarge the contact area between rod and soil. But how the current intensity affects on communication range is discuss as following.

Let us assume that the earth is a homogenous half space, according to theory of static current field, the difference of electric potential between point $\mathrm{P}$ and $\mathrm{Q}$ in Figure 2(a) can be represented as [8]

$$
\Delta V=\phi_{P}-\phi_{Q}=\frac{I}{2 \pi \sigma}\left[\left(\frac{1}{r_{P A}}-\frac{1}{r_{P B}}\right)-\left(\frac{1}{r_{Q A}}-\frac{1}{r_{Q B}}\right)\right],
$$

where $\phi_{P}, \phi_{Q}$ are the potential of point $\mathrm{P}$ and $\mathrm{Q}$ respectively, $\sigma$ is conductivity of the earth, and $r_{P A}, r_{P B}$, are the distances from $\mathrm{P}$ to rod (electrode) $\mathrm{A}$ and rod (electrode) $\mathrm{B}$ respectively, similarly $r_{Q A}$, and $r_{Q B}$ are the distance from $\mathrm{Q}$ to $\operatorname{rod} \mathrm{A}$ and $\operatorname{rod} \mathrm{B}$. Here we assume the two electrodes (A and B) are both single rod electrode.

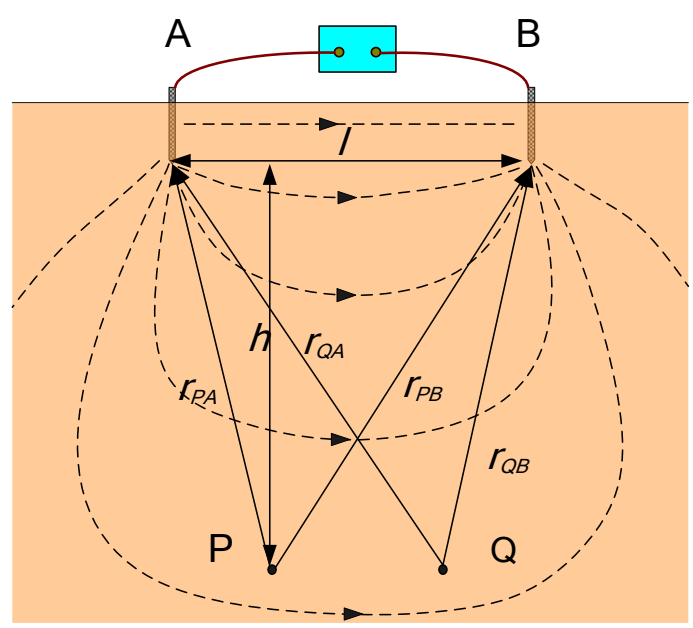

(a)

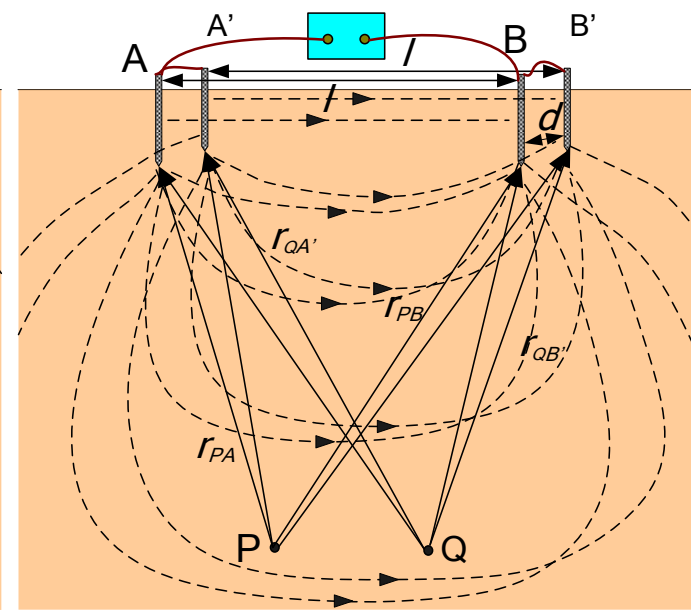

(b)

Figure 2. Schematic of Current Distribution in the Earth

If another rod (rod A' or rod B') is employed for each electrode with meters away from the first rod, and linked together with the first one by wire, as shown in Figure 2.(b). And the A-A'-B'-B is to be a rectangle, then the difference of potential between point $\mathrm{P}$ and $\mathrm{Q}$ will be

$$
\Delta V^{\prime}=\phi_{P}^{\prime}-\phi_{Q}^{\prime}=\frac{1}{2 \pi \sigma}\left[\left(\frac{I_{A}}{r_{P A}}+\frac{I_{A^{\prime}}}{r_{P A^{\prime}}}\right)-\left(\frac{I_{B}}{r_{P B}}-\frac{I_{B^{\prime}}}{r_{P B^{\prime}}}\right)\right]-\left[\left(\frac{I_{A}}{r_{Q A}}+\frac{I_{A^{\prime}}}{r_{Q A^{\prime}}}\right)-\left(\frac{I_{B}}{r_{Q B}}-\frac{I_{B^{\prime}}}{r_{Q B^{\prime}}}\right)\right],
$$

where $r_{X Y}$ is the distance between Point $X$ and $\operatorname{rod} Y$, such as $r_{P A}, r_{P A}, r_{P B}, r_{P B}, r_{Q A}, r_{Q A}$, $r_{Q B}, r_{Q B}, I_{Y}$ is the current intensity injected into the earth by $\operatorname{rod} Y$.

Generally the depth from the ground of the underground receiver $h$ is far longer than $d$ (the inter-electrode rods spacing, within $10 \mathrm{~m}$ as usual), $h \gg>d$, and hence $r_{A}>>d$, similarly, $r_{A}^{\prime}, r_{B}, r_{B}^{\prime}$ are all far longer than $\mathrm{d}$, then we can get $r_{P A} \approx r_{P A^{\prime}}, r_{P B} \approx r_{P B^{\prime}}$, $r_{Q A} \approx r_{Q A^{\prime}}$, and $r_{Q B} \approx r_{Q B^{\prime}}$, equation (2) then 


$$
\Delta V^{\prime}=\frac{1}{2 \pi \sigma}\left[\left(\frac{I_{A}+I_{A^{\prime}}}{r_{P A}}-\frac{I_{B}+I_{B^{\prime}}}{r_{P B}}\right)-\left(\frac{I_{A}+I_{A^{\prime}}}{r_{Q A}}-\frac{I_{B}+I_{B^{\prime}}}{r_{Q B}}\right)\right] .
$$

Because the volumes of current at two electrodes are equal, i.e., $I_{A 1}+I_{A 2}=I_{B 1}+I_{B 2}$, hence potential difference becomes

$$
\Delta V^{\prime}=\frac{I^{\prime}}{2 \pi \sigma}\left[\left(\frac{1}{r_{P A}}-\frac{1}{r_{P B}}\right)-\left(\frac{1}{r_{Q A}}-\frac{1}{r_{Q B}}\right)\right],
$$

where $I^{\prime}$ is the output current driven by the transmitter. By adding another rod for each electrode, the impedance between electrodes will be reduced, thus the injection current intensity increased, namely, $I^{\prime}>I$, hence it offers an improved potential difference between $\mathrm{P}$ and $\mathrm{Q}$, i.e., $\Delta V^{\prime}>\Delta V$. It indicates that with the potential difference improved, a receiver can detected the signal at a deeper spot with a same electrodes span. Therefore the communication range is enlarged.

If the $I^{\prime}$ is an AC current, i.e., $I^{\prime}=I_{m} \cos (\omega t+\theta)$, where $I_{m}$ is the amplitude of AC current. Owing to attenuation caused by skin effect, equation (4) should be multiplied by a attenuation factor $\left(1+\gamma h+\gamma^{2} h^{2}\right) e^{-\gamma h}$, then voltage amplitude between electrodes of receiver is given as

$\Delta V_{m}=\frac{I_{m}}{2 \pi \sigma}\left[\left(\frac{1}{r_{P A}}-\frac{1}{r_{P B}}\right)-\left(\frac{1}{r_{Q A}}-\frac{1}{r_{Q B}}\right)\right]\left(1+\gamma h+\gamma^{2} h^{2}\right) e^{-\gamma h}$.

Where $\gamma$ is the propagation constant given in [9], it is approximated by $\gamma \cong j \omega \mu \sigma, \omega$ is the angle frequency of signal, $\mu$ is the magnetic permeability of the earth. Suppose that $r_{P A}=r_{Q B}, r_{P B}=r_{Q A}$, then we have $r_{P A}=\sqrt{h^{2}+\left[\left(l-l_{P Q}\right) / 2\right]^{2}}$ and $r_{P B}=\sqrt{h^{2}+\left[\left(l+l_{P Q}\right) / 2\right]^{2}}$, where $l_{P Q}$ is the distance between $\mathrm{P}$ and $\mathrm{Q}$. Therefore the factors affect on communication depth can be summarized are as follows aspects:

(a) Current injected into the earth;

(b) Conductivity of earth medium;

(c) Electrodes span (both underground and on ground);

(d) Signal frequency.

According equation (5), a higher injection current intensity can result in a higher electrodes voltage in receiver, thus it can extend the communication depth. Multi-rods electrode scheme is a feasible and efficient way to minimize electrodes impedance and increase the current injected into the earth, but the impedance between multi-rod electrodes is also influenced by the number of rods, rods arrangement, rod buried depth (vertical depth), rod shape and materials. In this work, we mainly focus on rods number and rods arrangement, and their impacts on electrode impedance.

At very low frequency (ELF/ULF), the capacitive effect can be neglected, and the value of electrodes impedance is nearly equal to its real part: resistance. In our experiments, the frequency is at $22 \mathrm{~Hz}$, so the measured impedance value is considered as resistance.

\section{Experiments and Results}

The transmitting unit of TTE communication system with electrodes we established is shown in Figure 3. Processed by modulator (including coder and QPSK modulator), amplifier, and matched circuit, the data is then converted into a modulated sinusoidal current signal and injected into the earth through the electrodes. 
Two transceiver devices with different output power were developed for experiments, a surface transceiver with high power for on-ground test, and the underground device with low power for undermine experiment due to its safety demand. The underground transmitter device can be used on surface, as well as surface device can work in undermine if permitted. In coal mine well, the output power is strictly limited for safety requirements, therefore the output power of underground transceiver device is restricted within $5 \mathrm{~W}$. Both of surface device and underground device can transmit and receive signals in half-duplex mode.

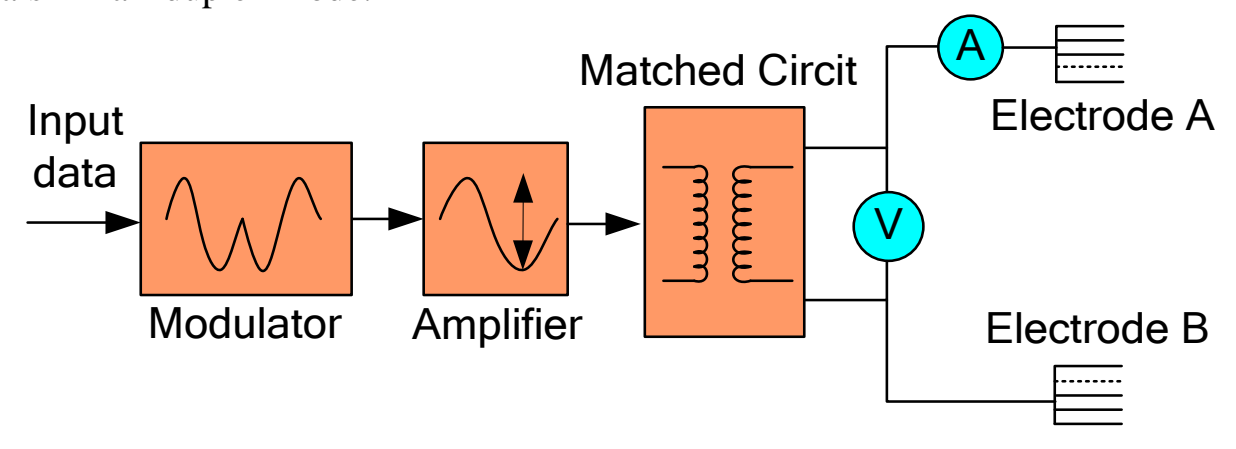

Figure 3. Schematic of Transmitter of TTE System with Electrodes

While signal transmitting, the output electrical voltage and current were measured by sampling the voltage and the current from the wires linked to electrodes, and then the sampled data was sent to a computer to process and calculate the output power.

The buried depth of rod can also affect the volume of electrodes resistance. Experimental results show that longer buried depth leads to lower electrodes resistance. The value of electrodes resistance with rod buried depth of $2 \mathrm{~m}$ is significantly less than that of $1 \mathrm{~m}$ depth or $1.2 \mathrm{~m}$ depth. The measured resistance values by our experiments we performed are shown in Figure 4. Measured data are obtained with electrode of 8-rods and 12 rods respectively. It illustrates the electrodes resistance decreases with buried depth increase.

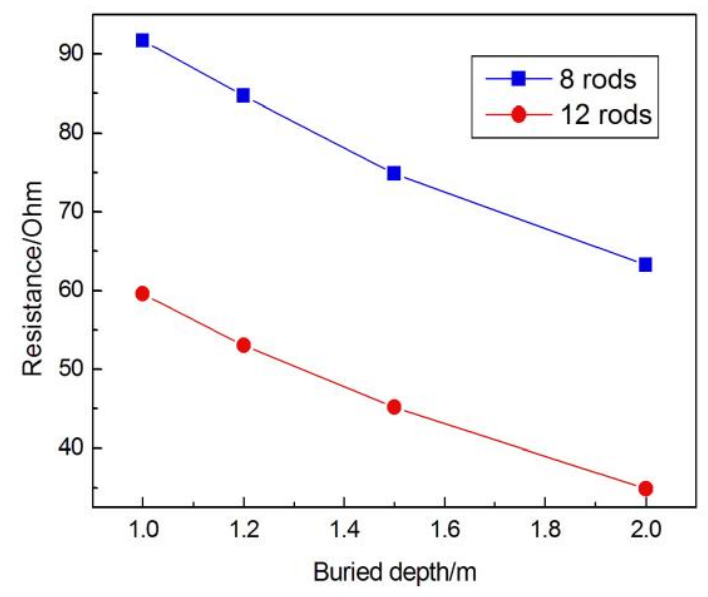

Figure 4. Measured Resistance Changing with Rod Buried Depth

Due to the difficulty for long rods in deploying, the steel rods we used in our experiments are $2 \mathrm{~m}$ in length, and $20 \mathrm{~mm}$ in diameter.

With aim to test the effects of rods number and rods arrangement on electrodes resistance, several experiments was performed on ground and in mine well. The first test 
spot is a coal mine area located in Qinyuan, Shanxi Province of China. Test spot's ground is of the classical loess plateau geomorphy, with surface medium of loess soil, and the underground medium in coal mine -- the roof over coal bed is mud stone with high humidity.

Three rods arrangement schemes were designed for on-ground test, as shown in Figure 5. In scheme 1, the rods in each group were arranged into a square with diagonal length of $6 \mathrm{~m}$, while in scheme 2 the rods were in a circle, with diameter of $6 \mathrm{~m}$. In scheme 3 , the rods of each electrode were deployed in a line, and it is the most possible arrangement in underground mine due to its narrowness. The distance between electrodes (electrodes span) is $60 \mathrm{~m}$ for all the three schemes.

Line arrangement is the unique scheme for underground test. Three rod groups (electrode A, B, C) were settled in different places of a roadway in mine well, with each rods group consisting of 11 rods deployed in a line with interval of $1.8 \mathrm{~m}$. The distance between group A and group B is $60 \mathrm{~m}$, while rod group $\mathrm{C}$ locates between group A and B with $40 \mathrm{~m}$ away from $\mathrm{A}$.
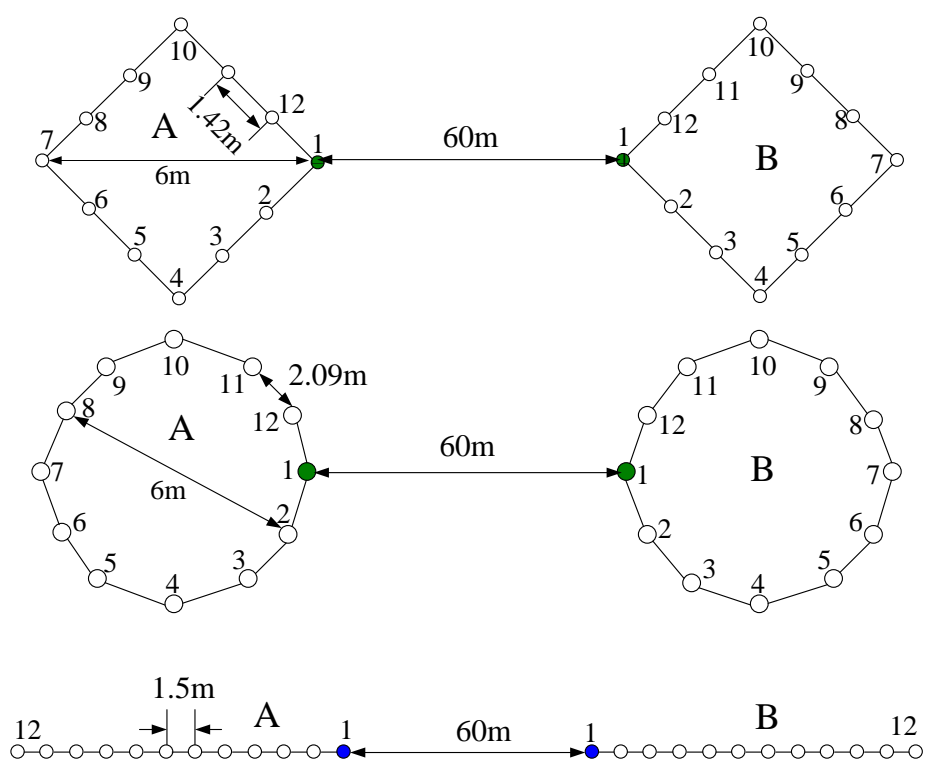

Figure 5. Schematic of Rods Arrangement Schemes

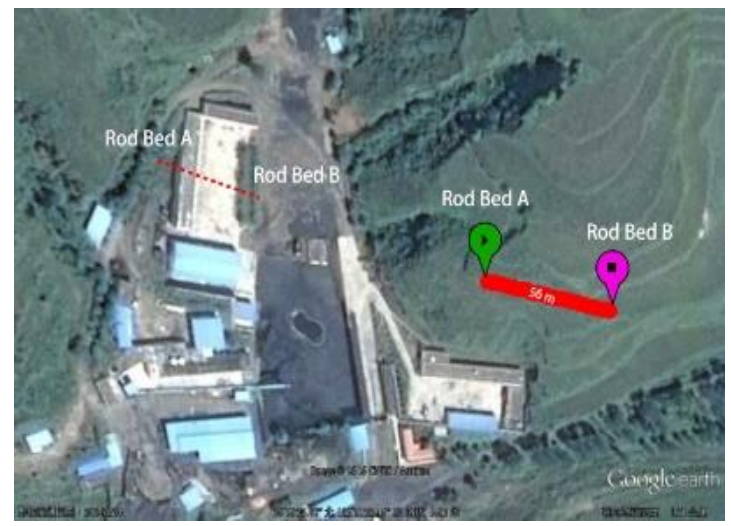

(a)

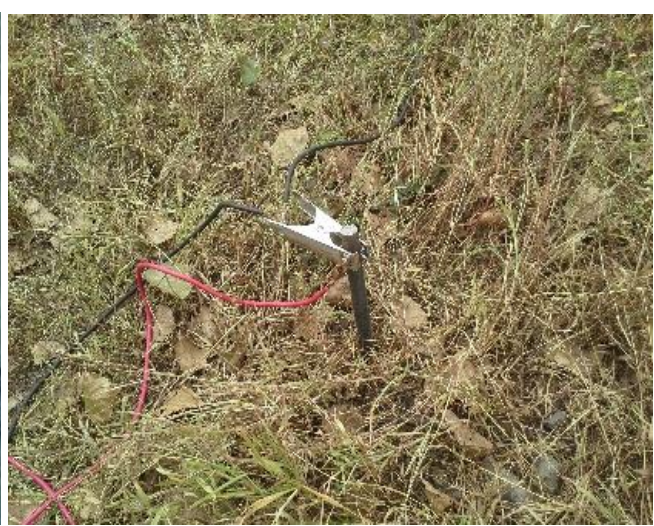

(b)

Figure 6. Test Spot Map and Steel Rods 
As Fig. 6 (a) shows, the test spot is on a flat hill top (the right rod beds, the left is the underground spot). In each rod bed, 20 steel rods with $20 \mathrm{~mm}$ in diameter and $2 \mathrm{~m}$-long were deployed into the earth to construct a 12-rod square rod group and a 12-rod circle rod group. The actual span between rod group A and group B is $56 \mathrm{~m}$ by measure. The line-arranged rod beds are in the lower adjacent terrace, with $55 \mathrm{~m}$ electrodes span by measure and parallel electrodes direction. Fig. 6 (b) shows the steel rod we used in our experiment.

Figure $7(a)$ is the on-ground experimental results. It illuminates that with the rods number increasing, the measured resistance values of three schemes decrease in coincidence. The measured electrodes resistance value of lined arrangement scheme is higher than that of both circled arrangement and squared arrangement with small size. Figure 7(a) also shows that with the rods number rising on, the slop of curve becomes flat and the resistance value is even nearly constant while rods number more than 10 , which means the electrodes resistance is minimized.

Underground test was performed in the main roadway tunnel at level -149 in DiBao Qinyuan coal mine well. Since there is no sufficient room for deploy square-arranged or circle-arranged rods in underground roadway, the rods were arranged in a line.

Fig. 7(b) shows the underground experimental results. We can see that with the rods number increase, the resistance values measured in 40m-span lined arrangement scheme and $60 \mathrm{~m}$-span lined arrangement scheme are decreased in same trend. With a certain rods number, the measured resistance of $60 \mathrm{~m}$ electrodes span is larger than that of $40 \mathrm{~m}$ span.

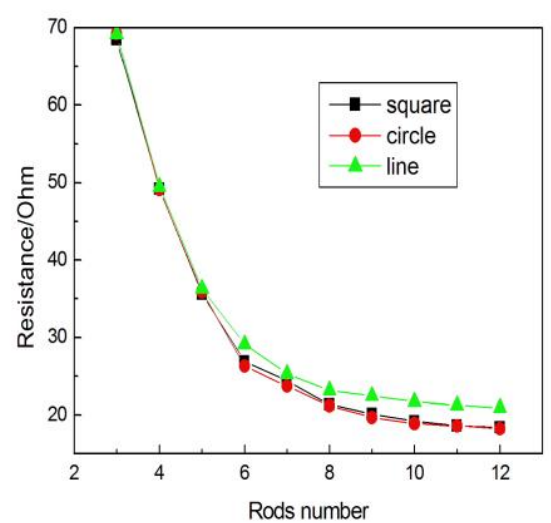

(a)

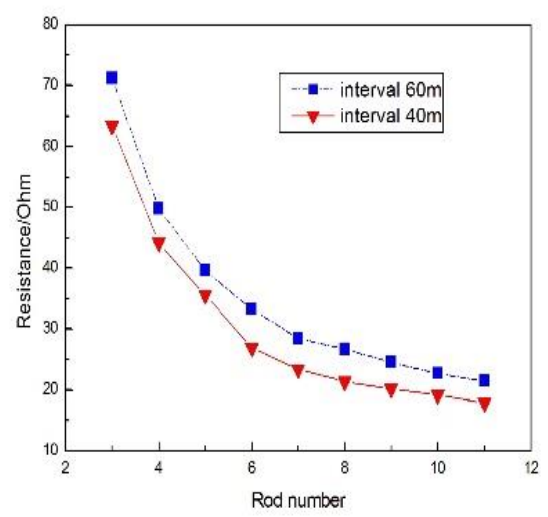

(b)

Figure 7. Comparison of Electrode Resistance Measured in Coal Mine

The test results of the signal receiving on the ground are given in Table 1. Because the data was spread by PN sequence in transmitter, the power level of received signal is lower than background noise and can not be measured correctly.

Table 1. Data Received or Not with Different Rods Number

\begin{tabular}{|l|l|l|}
\hline \multirow{2}{*}{$\begin{array}{l}\text { Rod } \\
\text { number }\end{array}$} & \multicolumn{2}{|c|}{ Signal received? } \\
\cline { 2 - 3 } & Span=40m & Span=60m \\
\hline 4 & No & No \\
\hline 5 & No & No \\
\hline 6 & No & No \\
\hline 7 & No & No \\
\hline 8 & No & Sometimes \\
\hline 9 & Sometimes & Yes \\
\hline 10 & yes & Yes \\
\hline 11 & Yes & Yes \\
\hline
\end{tabular}


Table I shows that the signal can be correctly received with transmitter electrodes span of $60 \mathrm{~m}$ and rods number of 8 , whereas it is received sometimes with same rods number but electrodes span of $40 \mathrm{~m}$. And actually the injection current intensity with $40 \mathrm{~m}$ electrodes span is higher than that with $60 \mathrm{~m}$ span as Fig. 7(b) indicates. That means a larger injection current intensity does not result in a longer communication distance if the electrodes span is reduced.

Figure 8.(a) shows the experimental results of underground and on-ground with same rod arrangement and electrodes span. We can see that the resistance values measured both underground and on the ground decrease with the rods number increase. And with same rods amount, the measured resistance value underground is a bit higher than that of on ground, the reason is the conductivity of underground mud rock is less than that of surface loess soil.

The electrodes resistance measured in copper mine roadway and on the ground are given in Figure 8 (b). The on-ground and underground test spots are in Hujiayu copper mine zone, Mount Zhongtiaoshan, Shanxi. The on-ground test spot is covered by semihumid drab soil, and the underground test spot -- roadway tunnel of underground mine is surrounded by humid granite porphyry. While the rods amount is less than 8 , the injection current to the earth in underground mine well is too weak and beyond range scope of our detection system. Even the rods number is up to $8,9,10,11$, electrode resistance values measured in underground test spot are significantly higher than measured on the ground test spot, and only rods number is up to 11, the signal sent by the high power transmitter (surface device) in underground roadway tunnel can be captured by the receiver on ground. The reason is that the conductivity of soil is far greater than that of quartz porphyry rock in mine roadway.

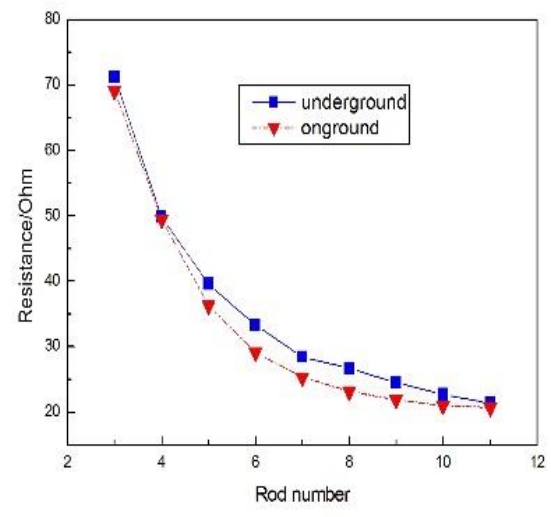

(a)

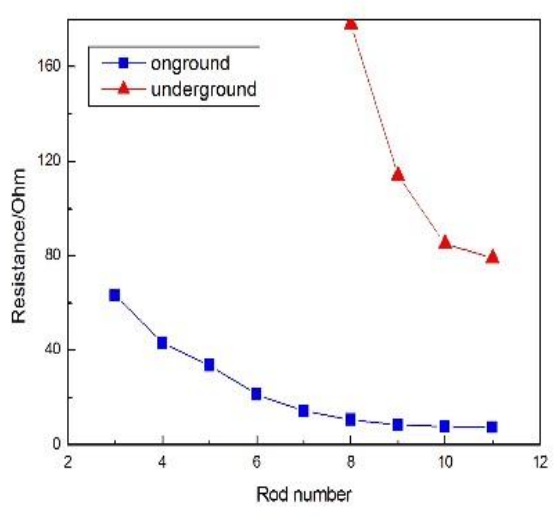

(b)

Figure 8. Comparison of Electrode Resistance Measured in Copper Mine

\section{Conclusion}

In spite of the sensitivity of receiver, the penetrating depth of TTE system is determined by the earth medium conductivities, the electrical current flows into the earth, electrodes span, and electrode contact resistance. A shorter electrodes span can lead to a larger transmitting power, but does not result in a longer penetrating depth.

In this paper, the experiments to achieve characteristics of electrodes resistances changing the rods number and rods arrangement have been performed at two different test sites. Although the measurements were made at different locations, results are in a consistent manner. The results show that the multiple-rods scheme can significantly reduce the electrodes resistance. And it also demonstrates that rods arranged in square or in circle are performing well than those in line in reducing resistance between electrodes. 
Experimental results also show the electrodes resistance is depending on depth of the rods buried, electrodes span, and the earth conductivity, which is more important in affecting transmitting power.

In short, for low frequency TTE communication system, multi-rod electrode scheme is an efficient way to evaluate its performance. Increasing number of rods and buried depth can minimize the electrodes impedance, whereas changing rod arrangement slightly affect the electrodes impedance.

\section{Acknowledgment}

This work is support by the project of Natural Science Foundation of China under Grant No. 61471224 and project of Natural Science Foundation of Shandong Province under Grant No. ZR2014JL044. Also the authors would like to thank for Jinfeng Qiao and Zhuorui Shen, from Shanxi Dibao Tech Co. Ltd, for their valuable works in the experiments.

\section{References}

[1] D. Gibson, "Channel Characterization and System Design for Sub-surface Communications", PhD Dissertation, University of Leeds, (2003); Leeds, U.K.

[2] J. Durkin, "Earth Conductivity Estimates From Through-the-Earth Electromagnetic Transmission Tests", IEEE Transactions on Geoscience and Remote sensing, vol. 29, no. 2, (1991), pp. 300-307.

[3] B. V. Munoz and A. Molina, "Earth impedance model for through-the-earth communication applications with electrodes", Radio Science, vol. 45, RS6015, (2010), pp. 807-812.

[4] V. Bataller, A. Munoz and N. Ayuso, "Channel Estimation in Through-The-Earth Communications with electrodes", Piers Online, vol. 7, no. 5, (2011), pp. 486-490.

[5] V. Bataller A. Munoz and P. M. Gaudo, "Electrode Impedance Measurement in through-the-earth Communication Applications", IET Microwaves, Antennas \& Propagation, vol. 6, no. 7, (2012), pp: 807-812.

[6] B. V. Munoz, A. Molina, P. Mediano, A. Mediano, J. A. Cuchí and J. L. Villarroel, "Improving medium access in TTE VLF-LF communications", Journal of Communications, vol. 4, no. 4, (2009), pp. 284294.

[7] L. Yan, J. A. Waynert and C. Sunderman, "Measurements and Modeling of through-the-earth Communications for Coal Mines,” IEEE Trans. Ind. Appl., vol. 49, no. 5, (2013), pp. 1979-1983.

[8] J. R. Wait, "Electromagnetic Surface Impedance for a Layered Earth for General Excitation", Radio Science, vol. 15, no. 1, (1980), pp. 129-134.
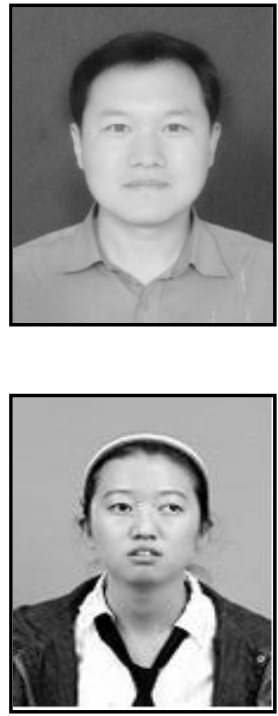

\section{Authors}

Jian Jun Hao is currently an associate professor of Shandong University of Science \& Technology, China. He received his M.S. and Ph.D. degree from China University of Ming and Technology (Beijing), Beijing, in 2004 and 2007, in information and communication engineering. His research interests include through-the-earth communication, signal processing and Wireless networks.

Yong Fei Mu was born in Shandong, China, in 1992. She received the B.S. degree from Qingdao University of Science and Technology (QUST), Qingdao, in 2014. She is currently studying for her M.S. degree in Signal and Information Processing in Shandong University of Science and Technology, Qingdao. Her research interests include communication network and information security. 


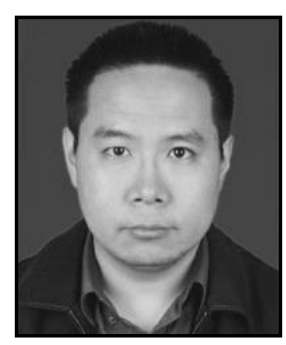

Bin Yan was born in Shandong, China. He received his $\mathrm{Ph} . \mathrm{D}$ degree from Harbin Institute of Technology (HIT) in 2007 in signal and information processing. He is currently an associate professor of Shandong University of Science \& Technology, China. His research and teaching interests include signal processing in wireless communication, security issues in multimedia communication. 